\title{
THE PRODUCTION OF EXPERIMENTAL PLAUT-VINCENT'S ANGINA IN THE DOG
}

\author{
By HELENE WALlACE, E. W. WALlACE and O. H. ROBERTSON
}

(From the Department of Medicine and the Department of Physiological Chemistry and Pharmacology, the University of Chicago, Chicago)

(Received for publication May 29, 1933)

Since the original description of Plaut-Vincent's angina some fifty years ago, a voluminous literature on this subject has accumulated. It is not the purpose of this paper to review the literature in any detail as this has been done elsewhere $(1,2)$. However, it may be stated briefly that the disease was described in 1883 by Miller (18), in 1894 by Plaut (3), and in 1896 by Vincent (4), as manifested by sore and bleeding gums, foul breath, and, in severe cases, by eventual ulceration of the mouth. They described the causative micro-organisms as a spirochete and a fusiform bacillus. Since then, these organisms have been found associated with pathological lesions in practically every part of the body, but especially in the upper digestive system, the lungs, and the genitalia.

Upon the isolation of the etiological agents the question was immediately raised as to whether they were separate entities or different forms of the same micro-organism. Weaver and Tunicliff (5) succeeded early in cultivating the fusiform bacillus from a patient with Vincent's angina, and on the basis of further work, Tunicliff (6) has stated that she believes the spirochete and bacillus are the same organism in different stages of a definite life cycle. Some workers (7) have supported her, but others (8), and in particular, Pratt (9), have come to the conclusion that these are two distinct micro-organisms.

The early literature stressed the infectious nature of the disease, but more recent observations indicate that the causative organisms are found in the normal mouth rather constantly and that the inception of infection depends on the presence of certain predisposing factors. Pratt found fusiform bacilli in 100 per cent of the two hundred people routinely examined at a large hospital. She also found the fusiform bacillus constantly and the spirochete occasionally in normal monkeys, guinea pigs, and rabbits. Other workers have reported analogous findings in these and other animals (2). Thus it would seem that the fusiform bacillus at least is present in the healthy mouth and is not in itself pathogenic. This is borne out by our work. Of forty dogs examined, thirty-eight showed the bacillus, and in those with poor teeth, spirochetes were usually present in the teeth pockets. ${ }^{1}$

${ }^{1}$ Vincent's angina is a recognized disease of the dog's mouth. 
Many attempts have been made to reproduce the disease experimentally. In 1923 Kline (10) produced abscesses in the traumatized groin of a guinea pig by injecting membranes from cases of Vincent's angina. Somewhat later Smith (11) succeeded in producing similar abscesses in the untraumatized guinea pig's groin by injection of material freshly obtained from human cases. He then injected into the lungs of rabbits pus from these experimental abscesses which showed spirochetes and fusiform bacilli in addition to other micro-organisms. Of ten rabbits infected, four developed lung abscesses, three gangrene, two pneumonia, and one, bronchiectasis. From certain of these lesions he isolated spirochetes and fusiform bacilli. Randall (12) injected scrapings from the gums of patients suffering from Vincent's angina into white rats, and Wagener (13), into kittens without success. Both workers used animals fed on a deficiency diet as well as normal ones. Wagener (13) traumatized the gums severely in some instances and made deep artificial pockets into which she injected the material, but the wounds healed and no fusiform bacilli or spirochetes could be recovered. Lichtenberg, Werner and Lueck (14) were unable to produce lesions in guinea pigs by the injection of pure cultures of fusiform bacilli into traumatized tissues, but did find spirochetes and fusiform bacilli in the membranes which formed over ulcers induced by injury of the mouth in normal guinea pigs.

While the above data indicate that experimental lesions of the groin and lung have been induced by the implantation of spirochetes and fusiform bacilli, the reproduction in animals of the clinical symptoms of Vincent's angina has not been successful.

In the spring of 1931 one of the authors (15) observed that a group of the dogs which he had been injecting daily with one of the squill glucosides, namely, scillaren $B$, developed a peculiar mouth condition similar to that found in Plaut-Vincent's disease. Smears from the mouths of these dogs showed the typical fusiform bacilli and spirochetes in large numbers. This observation suggested a possible method for the experimental production of Vincent's angina.

\section{METHODS AND MATERIALS}

Scillaren B, the drug used, is " the amorphous component of the natural mixture of the glucosides occurring in squill." ${ }_{2}$ It has the same action on the heart as digitalis, and possesses the cumulative properties of digitalis to the same degree. The toxicity on the mammalian heart is of the same order as that of ouabain. It was one of a number of squill glucosides used in a series of cumulative experiments on dogs (15); the others, scillaren A, scillicin, and crystalline scillonin, as well as ouabain ( $G$ strophanthin) and digitalis, did not produce the mouth effects noted with the possible exception of ouabain. One dog injected with ouabain developed typical mouth lesions, smears from which contained spirochetes and fusiform bacilli. The lesions healed spontaneously although the drug was not discontinued.

In the experiments just cited, a certain percentage (usually 25 per cent) of the lethal dose of the drug, measured in milligrams per kilo weight of the ani-

2 New and Non-Official Remedies, 1931. Our supply of scillaren B was obtained from the Sandoz Company, 708-710 Washington St., New York City. 
mal, was injected daily intravenously. The dogs injected with scillaren B were not run in a single series, but one or two at a time, simultaneously with other animals receiving different squill glucosides. A total of forty-eight dogs were used. Each drug was tested on eight dogs. Conditions of care, hygiene, and type of food were identical for all the dogs.

The dogs used in the present experiments were selected primarily for their good teeth and gums; they were all lively and in excellent condition. For the ease of handling, the small terrier type was chosen. Temperature and pulse were taken twice daily; weight and (in the case of five dogs) a white count, once a day. Detailed records, by means of sketches and notes, were made daily of the teeth, gums and mouth of each animal. At the same time, smears were taken from each side of the mouth, the incisors, and any suspicious pockets. The smears were stained by Tunicliff's method. ${ }^{3}$ All the animals were kept under excellent conditions and were well fed. The food given contained adequate amounts of the various vitamins.

A saline solution of the drug was injected daily into the antebrachial vein of the dog's foreleg. About 0.30 of the lethal dose was given for the first three days, and subsequently, 0.25 of the lethal dose until death. (The lethal dose as measured by the Hatcher-Brody cat method is $0.1 \mathrm{mgm}$. per kilo of body weight.) Photographs were taken of the dogs' mouths before the injection of the drug was begun, in various stages of the disease, and, in two cases, five minutes after death.

Autopsies were performed immediately after death. Particular attention was paid to the mouth, esophagus, stomach, trachea, lungs, heart, and kidneys. Tissues from ulcerating areas, as well as normal and only slightly affected regions, were excised and sectioned. These tissues were usually fixed in Zenker's acetic (in the case of the silver methods for spirochetes, 10 per cent neutral formalin was also used). The sections were for the most part stained with Giemsa's stain, prepared by Grübler, as this was found to stain both the spirochetes and fusiform bacilli distinctly, and also to give the best histological picture. Levaditi's silver method for spirochetes was also of value.

During the course of the disease in the animals, numerous cultures from the mouth were made.

\section{EXPERIMENTAL}

In the present study eleven dogs, six males and five females, were injected with scillaren B. All of them developed the characteristic lesions and bacteriological findings of Vincent's angina. In three dogs (4, 5 and 8 , Table I) the lesions were minimal, consisting of redness and sensitiveness of the gums with some bleeding, discoloration of the teeth, and the presence of numerous fusiform bacilli and some spirochetes. Two of these three animals, 5 and 8 , both of them very young dogs, died of an acute respiratory infection at this stage. ${ }^{4}$ Dogs $_{1}, 3,10$ and 11 exhibited a wellmarked clinical picture of the disease, with one or two ulcerations. Dogs 2, 6, 7 and 9 developed a severe form of the infection. In all these eight

${ }^{3}$ The stain, made up of $90 \mathrm{cc}$. of a saturated 95 per cent alcoholic solution of Grübler's gentian violet added to $10 \mathrm{cc}$. of 5 per cent phenol, was poured over the dried smears, and steamed for five minutes. The slides were then washed with tap water, blotted with filter paper, and examined under oil.

${ }^{4}$ Not fuso-spirochetal, as far as could be determined. 


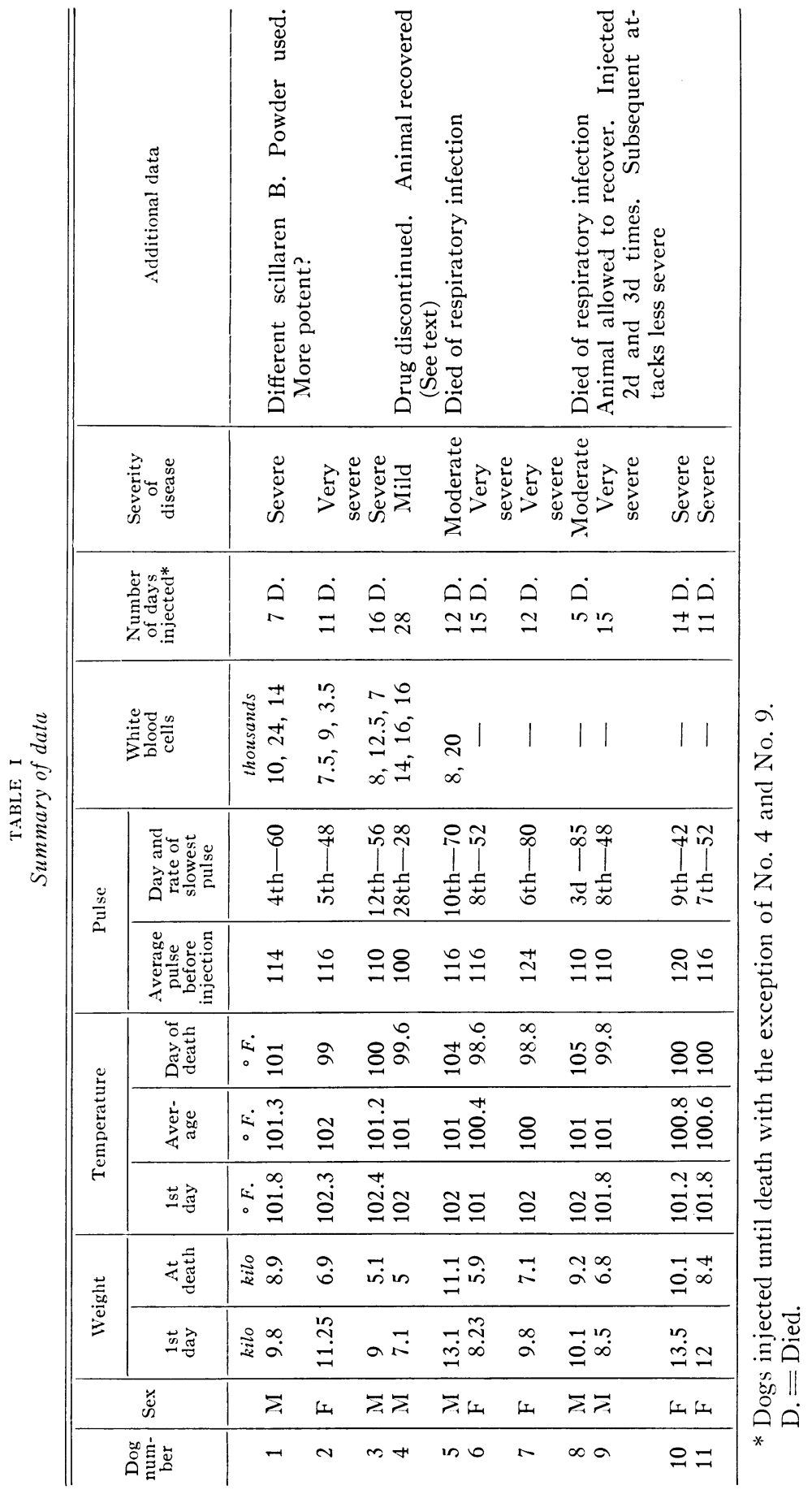


animals the molars became black, and the canines a very dark brown. Ulceration of various parts of the cheeks and tongue occurred. These ulcers were usually one to two centimeters in diameter. Several were considerably larger. There was much bleeding from the lesions.

As the disease progressed in a similar fashion in all the dogs, a general description may be made. During the first two days, the dogs were apparently normal, but after the injection on the third day, they all vomited profusely and were somewhat depressed. By this time smears from the mouth usually showed a decided increase in the number of small, short fusiform bacilli. By the fourth or fifth day vomiting was acute and very little food was eaten. The teeth, generally the posterior molars and canines, were somewhat discolored, the gum margins showed a narrow line of inflammation, and the smears from these regions contained quantities of short fusiform bacilli and a sprinkling of spirochetes. By the sixth day, the dogs were no longer lively or responsive, although the vomiting was not as acute. The gums were markedly inflamed and bleeding, the teeth darker, and the smears showed larger and more typical, cigar-shaped bacilli and also spirochetes. From the seventh to the fourteenth day the teeth became progressively clarker, until all but the incisors were a dark brown, the gums bled profusely and were very friable, and ulcers formed on the gums, cheek and tongue (Fig. 1). The smears showed large, typical, fusiform bacilli and spirochetes. Quantities of the latter were found wherever there was a bleeding and necrotic pocket.

During the injections the dogs lost a great deal of weight, in some cases almost half the original body weight There was no fever, but rather a drop in temperature during the last few days of the disease.

The white blood cell counts made on the first three dogs showed a decided increase at first, but one or two days before death the leukocytes dropped considerably, though not always below what might be considered normal. In Dog 4 the white count did not go down, but remained at a rather high level. This animal exhibited the mildest lesions in the series. Dog 5 , which died of a concurrent respiratory infection, showed a rise in the white blood cells to 20,000 . However, the leukocytosis apparently did not hinder the development of the Vincent's angina.

The animals died on an average about the fourteenth day in ventricular fibrillation. However, one $\log$ died as early as the seventh day, and another (Dog 4) did not succumb to twenty-eight injections. Most of the dogs were injected until they succumbed from the cumulative effects of the drug. In two animals the clrug was discontinued, as shown in Table I (Dogs 4 and 9). Dog 9 recovered from a severe attack of the experimental disease, and two months later, when a smear from the mouth showed only the few fusiform bacilli usually found in normal dogs, he was again injected, and developed a pronounced Vincent's angina as before. After a second recovery and a three month's interval, a third attack was induced. The second and third attacks were not as severe as the first. 
Table I is a summary of the general records on each dog, with the exception of the stained smears, which will be treated in more detail. All the animals exhibited similar changes in temperature, weight loss, etc., with the exception of Dog 4. This animal showed an increase in fusiform

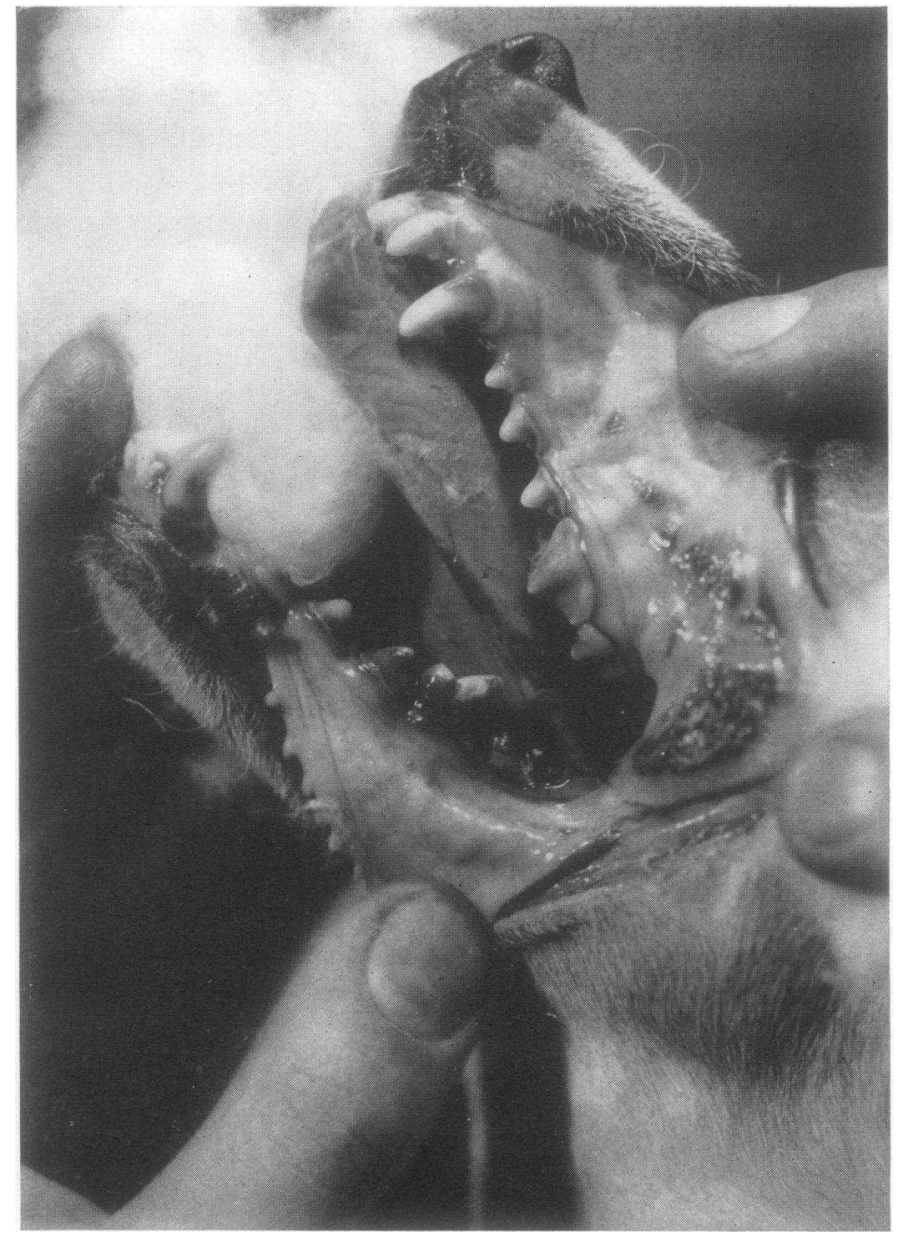

Fig. 1. Dog No. 6. Died on Fifteenth Day

Photograph, made 5 minutes after death, shows ulcerations of buccal mucosa and tongue, inflammation of gum margins and discoloration of teeth.

bacilli and spirochetes, and also developed a tender red margin on the gums with very slight bleeding, but the disease did not progress beyond this stage. He was injected for twenty-eight days (twelve days longer than any other animal) and the drug was then discontinued. The pulse at this time was only twenty-eight. The dog recovered completely and in two months was 
used for other experimental purposes. ${ }^{5}$ Dogs 5 and 8, the two animals that died of a respiratory infection, had a final temperature of 104 and $105^{\circ} \mathrm{F}$., respectively. However, early in the course of the disease their temperatures were normal.

As shown in the table, the average pulse rate for three days before injection was about 112. After the injection of the drug an initial progressive slowing of the heart occurred. Electrocardiograms taken at the time when the rate was slowest showed the heart to be in a condition of partial to complete block. Apparently the drug in this phase of its action progressively lengthened the conduction time until there was complete dissociation between auricular and ventricular contractions. The day on which the pulse was slowest generally occurred about the middle of the injection period. Later, as more toxic amounts of the drug accumulated, the heart rate increased. Apparently the ventricular musculature itself was stimulated. Ectopic foci appeared at various points in the ventricle, producing irregular QRS-T complexes of high potential and bizarre shapes. Before death the heart rate was usually too rapid to be counted.

\section{Attempted transmission}

Several control animals were kept in the room with the injected clogs and although they were allowed to eat out of the same pan as dogs with well advanced cases of Vincent's angina, they did not contract the disease. Swabs were then taken from the necrotic pockets in the gums of Dogs 6 and 7 and pushed into the gum margins of the control animals without any apparent effect, save that in one $\log$. whose physical condition was somewhat poorer than the others, there was some transient local redness and tenderness of the gums.

\section{Pathology}

At autopsy lesions were found only in the mouth. All the internal organs appeared normal. An extremely foul odor came from the mouth when it was opened. The teeth were badly discolored, the canines and molars being the most affected. Necrotic pockets filled with spirochetes were usually present at the base of these discolored teeth. In only one animal (Dog 3$)$ were the incisors affected. In this instance the molars were less involved than the incisors and the single ulcer present was at the base of the lower incisors. In Dogs 2, 6, 7 and 9 there were extensive cheek ulcers on both sides of the mouth. The first three of these dogs also had from one to five ulcers on the tongue, one to two centimeters long, and one-half to one centimeter wide. The largest ulcers were those on the cheek of $\operatorname{Dog} 7$. where the whole right sicle of the mouth was involved. All the ulcers, although irregular in shape. had well defined margins and

5 This $\log$ was subsequently found to be unusually resistant to infection with pneumococci and streptococci. 
in general were shallow, about three to four millimeters deep. They were approximately of the same depth throughout and only slightly excavated in the center.

Sections of the ulcers showed a superficial area of necrosis, beneath which the tissues were intensely infiltrated with cells, chiefly polymorphonuclear leukocytes. In the deeper parts of the inflammatory zone numerous large mononuclear cells were observed. The blood vessels were markedly engorged and in places the tissues were edematous. The necrotic area showed masses of bacteria composed principally of fusiform bacilli but containing also many cocci and other bacillary forms. In the layer of tissue immediately beneath the ulcerated surface fusiform bacilli were the predominating micro-organisms. They were observed not infrequently to be growing in large clumps and apparently in pure cultures. In this more superficial zone spirochetes were uncommon or absent, but in the deeper parts of the infiltrated tissue they were found in considerable numbers. Here the spirochetes were seen for the most part scattered through the tissue, but in places they were observed to be growing in colonies, some of which were of considerable size and apparently free from other microorganisms. The number of fusiform bacilli present in the tissues diminished progressively with the distance from the surface, and they were often not found in the areas invaded by the spirochetes. While the sections showed in general this separation of the fusiform bacilli and spirochetes, there were areas where both were present, notably within the muscle bundles. Here the micro-organisms were observed lying parallel, as if between the individual fibers. In places the muscle fibers had apparently disappeared, leaving somewhat wavy bundles of spirochetes, fusiform bacilli, or both together. Toward the distal extremity of the muscle bundle the spirochetes predominated. Cocci and short bacilli (non-fusiform) were distributed irregularly through the tissue. They were most abundant near the surface and rare or absent in the depth of the inflammatory area. In one animal (Dog 2) long filamentous fusiform bacilli were found growing in great numbers in a section of gum ulcer.

The reaction of the tissues and the distribution of the micro-organisms observed in the lesions of the dog's mouth resemble substantially the histological findings in the diseased tissues of human cases of Vincent's angina (16).

\section{Bacteriology ${ }^{6}$}

The so-called typical smear of Vincent's angina was not seen until ne-crosis had occurred. Before necrosis appeared the fusiform bacilli far outnumbered the spirochetes. Practically all of the fusiform bacilli seen in the smears of the first three days were small. The large cigar-shaped

${ }^{6}$ The work on cultivating the organism was done in collaboration with Miss Helen Van Sant of the Department of Medicine. 
fusiform bacilli only became numerous about the sixth day, at which time spirochetes and necrosis also appeared. The spirochetes were found in abundance in the pockets and necrotic regions (Fig. 2). The "typical"

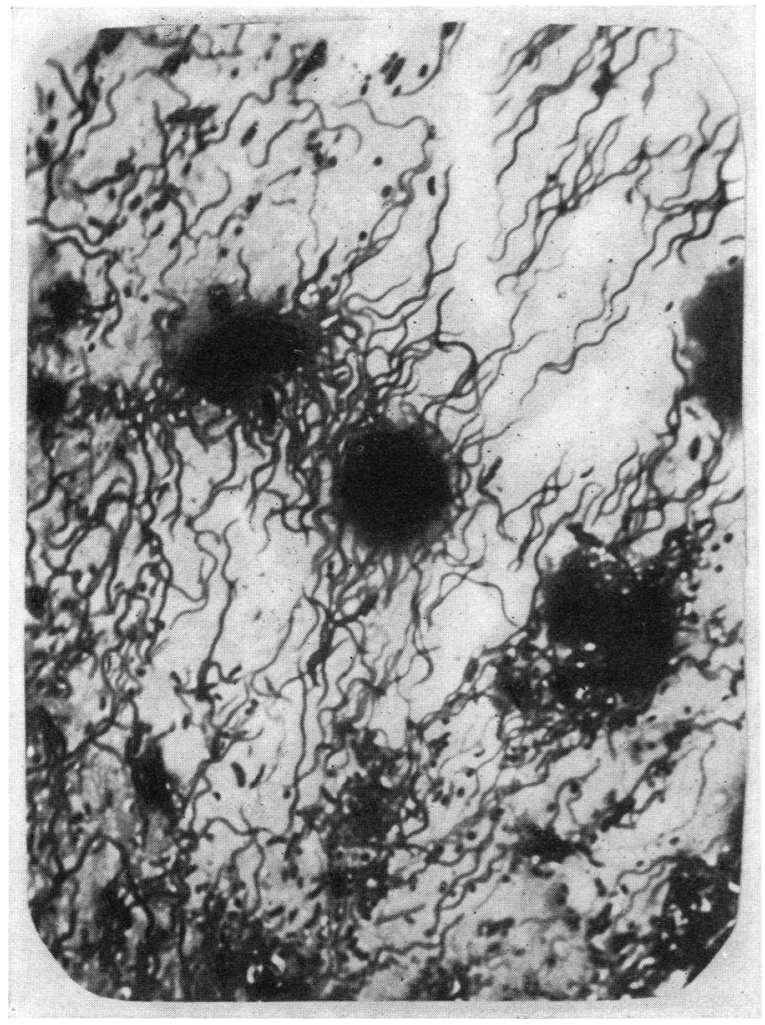

FIG. 2. Dog No. 9

Smear taken from necrotic pocket in gum on tenth day of disease shows nasses of spirochetes. $1200 \times$.

Vincent's angina smear (Fig. 3) could be obtained at the end of the first week of injection and at any subsequent time. In this microphotograph the spore-like character of the fusiform bacilli may be seen. Long filaments of fusiform bacilli were not uncommonly observed (Fig. 4). In some cases they resembled the filamentous forms seen in the cultures and sections. The smears at this time showed practically no other organisms than the fusiform bacilli and spirochetes.

A study of a number of human cases of Vincent's angina gave analogous findings. They showed all the variations of the fusiform bacilli that were present in the dogs' mouths. Likewise in the human cases the spirochetes were found in the teeth pockets or deep in the necrotic tissue, such as occurred in tonsillar crypts. 


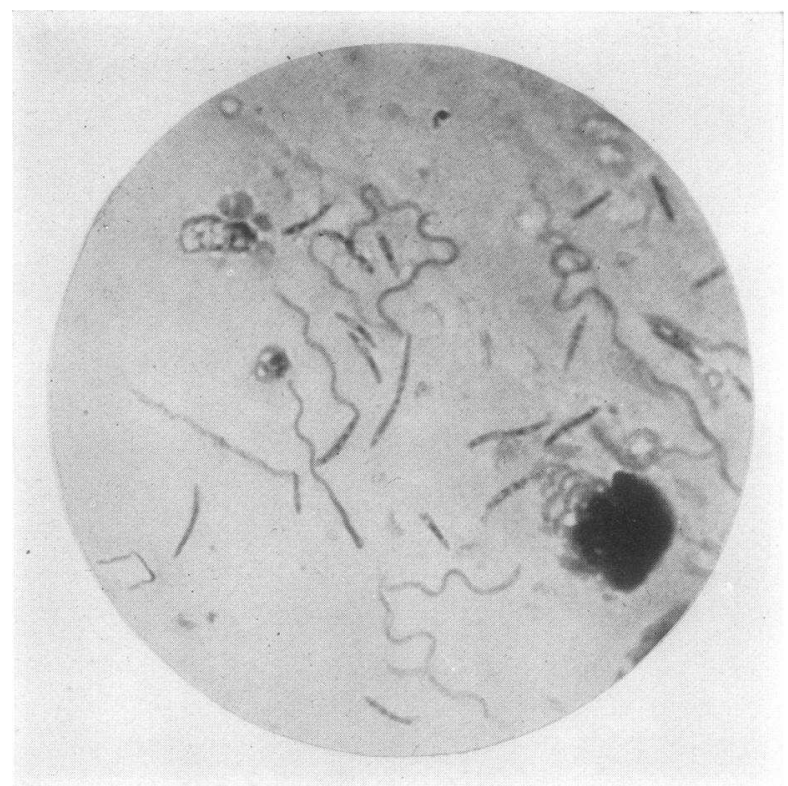

FIG. 3. Dog No. 6

Smear made from gum on ninth day of disease shows typical spirochetes and fusiform bacilli of Vincent's angina. $1200 \times$.

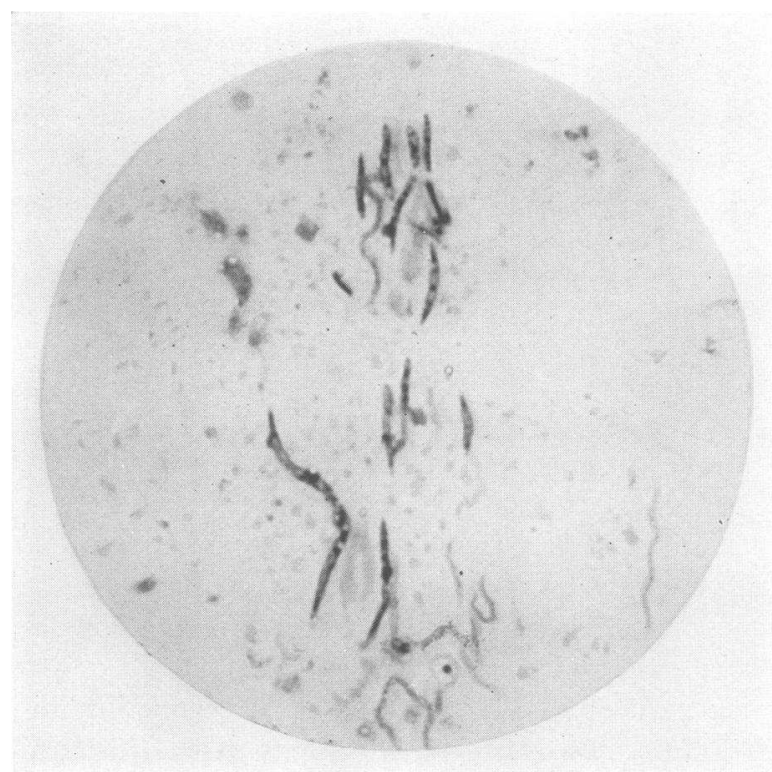

Fig. 4. Dog No. 7

Smear made from gum on eighth day of disease, showing long filamentous and short cigar-shaped forms of fusiform bacilli. $1200 \times$. 
Cultures were taken from the dogs' mouths when the smears showed many spirochetes and fusiform bacilli. A number of cultures were also made from the pockets about the teeth, ulcers, and regions where the smears showed a predominance of one micro-organism or the other. Most of the cultures were made with material from Dog 9.

Various culture media were used. The fusiform bacilli grew fairly well on rabbit's blood agar (one part of the blood to three parts of beef infusion agar, $\mathrm{pH} 7.4$ to 7.6 ), but not nearly as readily as on ascitic agar (one part of ascitic fluid to three parts of a beef infusion agar, $\mathrm{pH} 7.4$ to 7.6). Ascitic agar and a beef infusion-ascitic broth (three parts of one per cent dextrose beef infusion broth to one part ascitic fluid, $\mathrm{pH} 7.4$ to 7.6) were our most satisfactory media. With Loeffler's medium, recommended by Tunicliff, we were less successful. We also used Noguchi's leptospira medium (17) in an attempt to grow the spirochetes.

All the cultures were grown anaerobically. Although we tried anaerobic plates as well as anaerobic jars, in which the air had been evacuated and replaced with $\mathrm{CO}_{2}$ or nitrogen, we found that Wright's anaerobic method, besides being the simplest, was also the best for our purpose. Test tubes were stoppered by cotton saturated with a five per cent $\mathrm{NaOH}$ solution. A small amount of pyrogallic acid was placed on top of this, and the tube corked, and sealed with paraffin. Incubating temperature was $37^{\circ} \mathrm{C}$.

We were able to isolate colonies of the fusiform bacilli quite readily after forty-eight hours incubation. They were minute and, while not absolutely transparent, they seemed so when contrasted with the opaque colonies of cocci which formed the bulk of the growth. Single colonies were transferred to ascitic agar slants and were maintained in pure culture without difficulty. Transplants to fresh media were made every three days for two weeks, and subsequently, every two weeks for three months. After some months the bacillary forms became more or less degenerate although a slant examined eleven months subsequent to the initial culture still contained some recognizable fusiform bacilli. At no time were we able to cultivate the spirochetes-even when the inoculum was obtained from pockets showing practically a pure growth of spirochetes; nor did we find spirochetes at any time in our cultures of fusiform bacilli. Subcultures of fusiform bacilli in leptospira medium yielded only fusiform bacilli.

The fusiform bacilli in the pure cultures were small at first, but grew rapidly to the usual size, and subsequently, into the long filamentous forms, which at times stretched completely across the microscopic field. They were also often wavy, and to the casual observer might be taken for spirochetes. They were, however, much larger than the Vincent's spirochete and under lark field examination were seen to be non-motile.

As a rule, once the fusiform bacilli had attained the filamentous stage, they showed no further change. Occasionally small fusiform bacilli appeared in cultures in which only filamentous forms had been observed for 
a week. The long filamentous forms of the cultures are exactly similar, morphologically at least, to those seen in the sections; both are dotted at more or less regular intervals. The long bacilli of the smears which apparently were made up of a number of bacilli joined end to end, were much thicker than those seen in the cultures and sections.

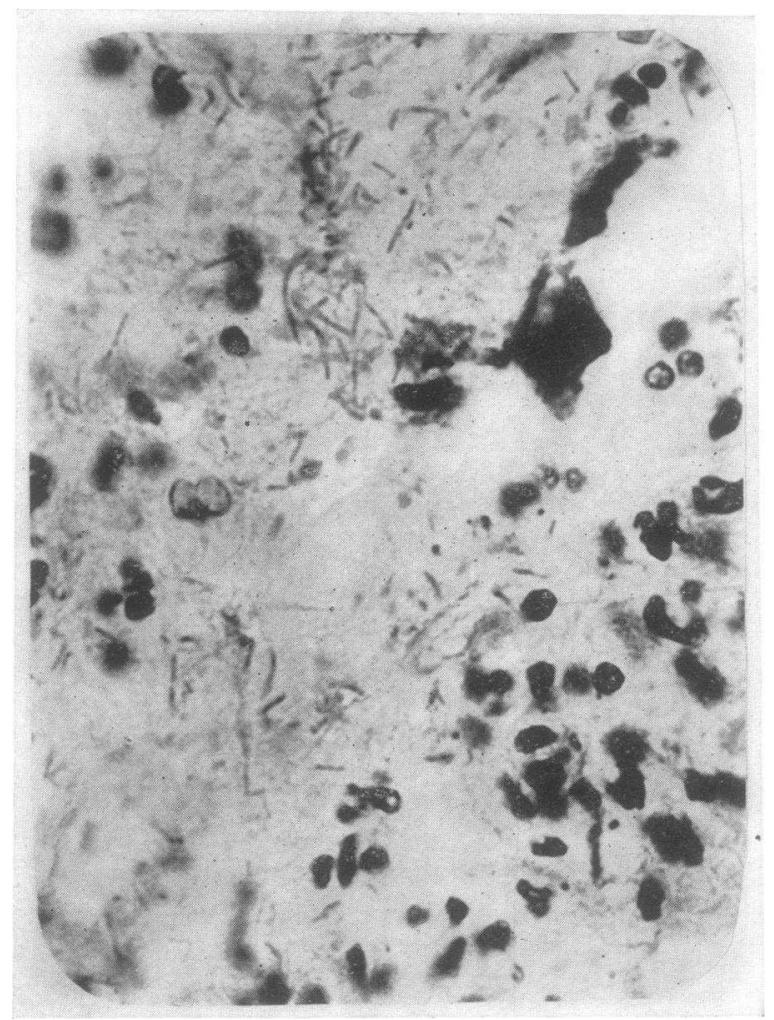

Fig. 5. Dog No. 6. Section of Tongue Ulcer. Photograpil Made of the More Superficial Zone of the Lesion

Shows many fusiform bacilli and an occasional spirochete. $1200 \times$.

Scillaren B solution was added in various amounts to the different media, but the organisms grew better without it even though the amount added was minute.

\section{DISCUSSION}

We have no explanation to offer concerning the mechanism by which the drug scillaren B acts to induce the lesions which we have described in the dog's mouth. From our present but very incomplete knowledge of those conditions which are considered to favor the spontaneous occurrence of Vincent's angina in animals and human beings, namely poor nutritional states due to unbalanced diets, chronic disease, etc., it would seem possible 
that the injection of scillaren B disturbs metabolic processes in such a manner as to lower the resistance of the buccal mucous membrane to the spirochetes and fusiform bacilli normally present. Whether this deleterious effect of the drug is principally a generalized or a local one we do not know. While it is true that the animals all showed marked gastro-intestinal disturbances and an associated loss in weight, these same effects were produced to an equal degree by the other squill derivatives employed but without the subsequent development of fuso-spirochetal mouth lesions except in one instance. Thus it would seem that either the general effect of scillaren $\mathrm{B}$ is of a peculiar nature or that it produces a characteristic local disturbance not shown by the five other closely related compounds. The excretion of the clrug locally in the mouth cannot be ruled out as a cause of the lesion, but neither can it be determined as such since the amounts injected are far too small to render recovery of the drug possible. It is of course conccivable that the production of the lesions in the mouth by this one squill glucosicle is due to impurities in the preparation rather than to the compound itself. Of two lots of scillaren B used, one was found to cause the development of the experimental disease much more quickly than the other.

The data presented inclicate that a leukopenia is not a factor in favoring inception of the disease nor does a leukocytosis appear to influence its course.

Our unsuccessful attempts to transmit the disease to healthy dogs, while too few in number to permit conclusions, suggest that the fusiform bacilli and spirochetes may not be pathogenic for the normal animal, even when applied to the gums and mucous membranes of the mouth in large numbers. In a recent paper, Lichtenberg and his co-workers (14) question the pathogenicity of these micro-organisms for normal human beings. However, there are well authenticated reports in the literature of small epidemics of Vincent's angina among normal persons living under apparently healthful conditions. This leads one to infer that either the normal dog possesses a greater natural resistance to the fuso-spirochetal micro-organisms than do human beings, or that the strain found commonly in the dog differs in pathogenicity from that occurring in the human mouth.

With the evidence available we feel that it is not yet possible to draw definite conclusions concerning the identity or non-identity of the spirochete and fusiform bacillus. Our cultural studies do suggest that these are two distinct microbial forms. If the fusiform bacillus and the spirochete represented different phases of a single life cycle one might expect that in a culture medium highly favorable to the multiplication of the bacillary form, the spirochetal phase would develop. However, since we have not been able to contrive sufficiently favorable conclitions in the test tube for the growth of spirochetes freshly isolated from the lesions, we have no means of knowing whether or not spirochetes would evolve from fusiform bacilli were a suitable growth medium provided. 
SUM MARY

By means of a daily intravenous injection of 0.25 lethal dose of scillaren B, a squill glucoside, it was found possible to produce the typical clinical picture of Plaut-Vincent's angina in the dog. The first manifestations of the experimental disease occurred on the fourth or fifth day with redness and bleeding of the margins of the gums and an associated discolorization of the teeth. The inflammation rapidly became more intense, spreading to the cheeks and tongue where ulcers often developed. When the drug was continued death occurred on the average about the fourteenth day. In certain $\operatorname{logs}$ the injections were discontinued and the animals recovered. Smears from the lesions in the mouth showed the characteristic fusiform bacilli and spirochetes found in human beings suffering from Vincent's angina.

Sections of the necrotic tissues and ulcers contained great numbers of fusiform bacilli and spirochetes. The former were present chiefly in the superficial zone of the inflammatory area; the latter were confined largely to the deeper part of the lesion.

Pure cultures of the fusiform bacilli were grown from material obtained from the dogs' mouths. These organisms varied from short bacilli in young cultures to long filamentous forms in older ones and were nonmotile. Attempted cultivation of the spirochetes was not successful.

\section{BIBLIOGRAPHY}

1. Harrell, V., Arch. Otolaryng., 1931, xiv, 1. The Present Status of PlautVincent's Infection.

2. Smith, David T., Oral Spirochaetes and Related Organisms in Fuso-Spirochaetal Disease. Williams and Wilkins Co., Baltimore, 1932.

3. Plaut, H. C., Deutsche med. Wchnschr., 1894, xx, 920. Studien zur bacteriellen Diagnostik der Diphtherie und der Anginen.

4. Vincent, H., Ann. Inst. Pasteur, 1896, x, 488. Sur l'Étiologie et sur les Lésions Anatomo-Pathologiques.

Vincent, H., Bull. et mém. Soc. méd. d. hôp. de Paris, 1898, xv, 244. Sur une Forme Particulière D'Angine Diphtéroide.

5. Weaver, G. H., and Tunicliff, R., J. Infect. Dis., 1905, ii, 446. The Occurrence of Fusiform Bacilli and Spirilla in Connection with Morbid Processes.

6. Tunicliff, R., J. Infect. Dis., 1906, iii, 148. The Identity of Fusiform Bacilli and Spirilla.

Ibid., 1923, xxxiii, 147. The Life Cycle of Bacillus Fusiformis.

7. Larson, W. P., and Barron, M., J. Infect. Dis., 1913, xiii, 429. Report of a Case in which the Fusiform Bacillus Was Isolated from the Blood Stream.

Klimenko, W. N., Centralb1. f. Bakt., 1914, 1xxiv, 487. Die Bedeutung der Spindelbacillen in der Pathologie des Sharlachs.

8. Krumwiede, C., Jr., and Pratt, J. S., J. Infect. Dis., 1913, xiii, 438. Fusiform Bacilli: Cultural Characteristics.

Varney, P. L., J. Bact., 1927, xiii, 275. The Serological Classification of Fusiform Bacilli. 
9. Pratt, J. S., J. Infect. Dis., 1927, xli, 461. On the Biology of B. Fusiformis.

10. Kline, B. S., J. Infect. Dis., 1923, xxxii, 481. Experimental Gangrene.

11. Smith, D. T., J. Infect. Dis., 1930, xlvi, 303. Fusospirochetal Disease of the Lungs Produced by Cultures from Vincent's Angina.

12. Randall, S. J., J. Dent. Research, 1931, xi, 49. An Attempt to Produce Vincent's Infection in the White Rat.

13. Wagener, E. H., et al., J. Dent. Research, 1930, x, 591. The Laboratory Diagnosis of Vincent's Infection.

14. Lichtenberg, H. H., Werner, M., and Lueck, E. V., J. A. M. A., 1933, c, 707. The Pathogenicity of the Fusiform Bacillus and Spirillum of Plaut-Vincent.

15. Wallace, E. W., and Van Dyke, H. B., pending pub. J. Pharmacol. and Therap., 1933. Cumulative Poisoning by Squill Derivatives and by Ouabain.

16. Kolle, W., Kraus, R., and Uhlenhuth, P., Handb. d. Path. Mikro-organismen, 1928, Volume V, Part I, 607. "Bacillus fusiformis" (By H. A. Gins).

17. Noguchi, H., “The Spirochaetes.” Newer Knowledge of Bacteriology and Immunology. University of Chicago Press, 1928, p. 452.

18. Miller, W. D., N. England J. Dent., 1883, ii, 229. Further Contributions on the Subject of Dental Caries. (Quoted by Harrell (1).) 